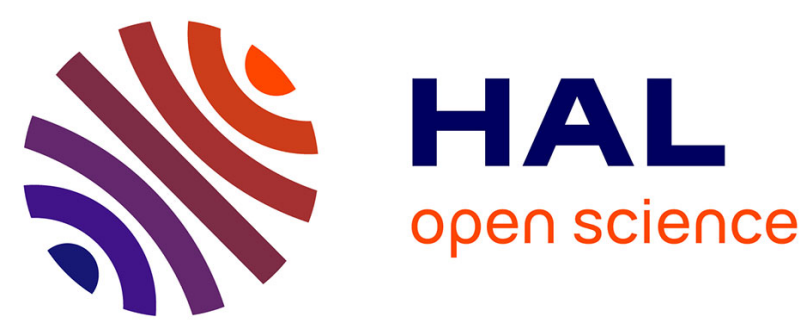

\title{
Low-frequency dynamics of water absorbed in Nafion membranes as a function of the temperature.
}

Alessandro Paciaroni, Mario Casciola, Elena Cornicchi, Matteo Marconi, Giuseppe Onori, A. Donnadio, M. Sganappa, Alessio de Francesco

\section{To cite this version:}

Alessandro Paciaroni, Mario Casciola, Elena Cornicchi, Matteo Marconi, Giuseppe Onori, et al.. Low-frequency dynamics of water absorbed in Nafion membranes as a function of the temperature.. Philosophical Magazine, 2007, 87 (3-5), pp.477-483. 10.1080/14786430600930200 . hal-00513757

\section{HAL Id: hal-00513757 \\ https://hal.science/hal-00513757}

Submitted on 1 Sep 2010

HAL is a multi-disciplinary open access archive for the deposit and dissemination of scientific research documents, whether they are published or not. The documents may come from teaching and research institutions in France or abroad, or from public or private research centers.
L'archive ouverte pluridisciplinaire HAL, est destinée au dépôt et à la diffusion de documents scientifiques de niveau recherche, publiés ou non, émanant des établissements d'enseignement et de recherche français ou étrangers, des laboratoires publics ou privés. 


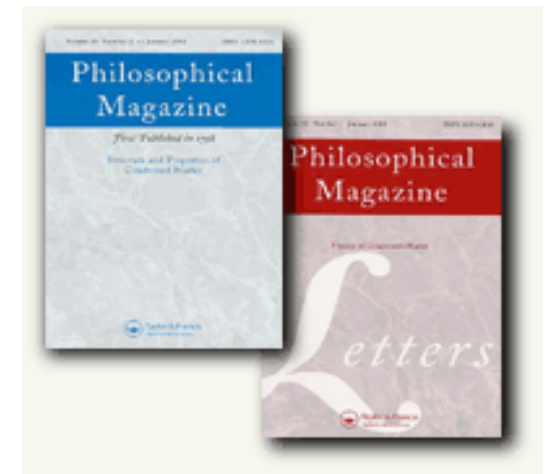

\section{Low-frequency dynamics of water absorbed in Nafion membranes as a function of the temperature.}

\begin{tabular}{|r|l|}
\hline Journal: & Philosophical Magazine \& Philosophical Magazine Letters \\
\hline Manuscript ID: & TPHM-06-Apr-0122.R1 \\
\hline Journal Selection: & Philosophical Magazine \\
\hline Author: & $26-$ Jul-2006 \\
\hline & $\begin{array}{l}\text { Complete List of Authors: } \\
\text { Marconi, Matteo; University of Perugia, Physics } \\
\text { Casciola, Mario; University of Perugia, Chemistry } \\
\text { Donnadio, A.; University of Perugia, Chemistry } \\
\text { Sganappa, M.; University of Perugia, Chemistry } \\
\text { De Francesco, Alessio; University of Perugia, Physics }\end{array}$ \\
\hline Keywords: & neutron, polymers \\
\hline Keywords (user supplied): & water, dynamical transition, dynamics \\
\hline &
\end{tabular}

\section{S scholaroNE" \\ Manuscript Central}




\section{Low-frequency dynamics of water absorbed in Nafion membranes as a function of the temperature.}

A. Paciaroni ${ }^{*}, 1,2$, M. $_{\text {Casciola }}^{2,3}$, E. Cornicchi ${ }^{1,2}$, M. Marconi $^{1,2}$, G. Onori $^{1,2}$,

A. Donnadio ${ }^{2,3}$, M. Sganappa ${ }^{2,3}$, A. De Francesco ${ }^{4}$

${ }^{1}$ Dipartimento di Fisica dell'Università di Perugia, and CNR-INFM CRS SOFT, Via A. Pascoli, I06123 Perugia, Italy.

${ }^{2}$ CEMIN (Centro di Eccellenza per i Materiali Innovativi e Nanostrutturati), c/o Dipartimento di Chimica dell'Università di Perugia, Via Elce di Sotto 8, I-06123 Perugia, Italy.

${ }^{3}$ Dipartimento di Chimica dell'Università di Perugia, Via Elce di Sotto 8, I-06123 Perugia, Italy.

${ }^{4}$ CNR-INFM CRS SOFT, I-00185 Roma, Italy c/o Institut Laue-Langevin, Rue Jules Horowitz 6, F-38042 Grenoble, France.

We performed a neutron scattering study to investigate the dynamical behavior of water absorbed in Nafion at low hydration level $(\lambda=6, \lambda=$ moles of water/moles of sulfonic acid sites) as a function of temperature in the range $200 \mathrm{~K} \div 300 \mathrm{~K}$. To single out the signal of the confined water the measurements were done on samples hydrated with both $\mathrm{H}_{2} \mathrm{O}$ and $\mathrm{D}_{2} \mathrm{O}$ in the same temperature range. Due to the strong incoherent scattering cross section of hydrogen atoms with respect to deuterium, in the difference spectra the contribution from the Nafion membrane is subtracted out and most part of the spectra originates from absorbed water. The estimated dynamical susceptibility exhibits features that resemble those of bulk water. In particular, the spectra display a bump at around $1 \mathrm{meV}$, possibly related to the $\alpha$ relaxation, the intensity of which is markedly affected by the temperature change. Two features due to the phonon-like collective hydrogen bond network dynamics are well visible at approximately $7 \mathrm{meV}$ and $25 \mathrm{meV}$.

Keywords: Confined water; dynamical transition; $\alpha$ relaxation; fuel cells; neutron scattering 


\section{Introduction}

The dynamical properties of water when it is confined within hydrophobic or hydrophilic boundaries are remarkably different from those of bulk water [1-4]. In particular, the behaviour of water confined in nanometer-sized volumes has drawn the attention of several studies over the last decades, in view of its central importance in biological, geological and technological contexts $[5,6]$. In spite of the large number of experimental data collected in the past, the microscopic mechanisms that rule the confined water dynamics have not yet been fully understood. In polymer electrolyte membranes, such as Nafion, it is crucial to characterise the water dynamical behaviour as hydration is essential for proton conduction processes [7,8]. The Nafion membrane shows high proton conductivity and exceptional mechanical, thermal and chemical stability, then it if often employed in fuel cells [9]. There is general consensus that these favourable characteristics result from its nanophase-segregated structure in which hydrophilic clusters are embedded in the hydrophobic matrix. However the structure of the membrane itself is still a matter of discussion $[8,10,11]$. In the well-known model of Gierke et al. [10], the ionomer polar heads (sulfonic groups) form nanometersized spherical aggregates, where water molecules would cluster in nano-pools, connected by small channels. Conversely, in the very recent elongated polymer particle model [11], where the Nafion matrix is supposed to be made of elongated (cylindrical or ribbon-like) polymeric aggregates, surrounded by ionic groups and collinearly packed in bundles, that are themselves randomly oriented in space at the sub-micron scale. In this model the water molecules form a continuous solvation phase around the polymeric aggregates.

Because of the exceptionally large incoherent scattering cross section of hydrogen, neutron scattering is an attractive technique for studying the dynamics of water confined in Nafion membranes. In recent quasielastic neutron scattering investigations it has been found that the dynamics of protons in low-hydrated Nafion can be schematised as a random jumping within a confining sphere, whose size suggests that diffusion takes place effectively in a restricted region around the sulfonic hydrophilic groups [12]. Therefore the emerging picture is that of a tightly coordinated water, consistent with NMR [13] and differential scanning calorimetry experiments [14] that indicate that at low water content, below around $0.08 w$ ( $w=$ weight of water/weight dry Nafion), only strongly and loosely bound water would exist, which may be supercooled down to sub-zero temperatures.

Here we present the results of a neutron scattering investigation performed to study the lowfrequency features of water absorbed in Nafion as a function of the temperature in the range from $200 \mathrm{~K}$ to $300 \mathrm{~K}$. In the low-frequency range around $1 \mathrm{meV}$ the calculated dynamical susceptibility exhibits a noticeable increase up to $260 \mathrm{~K}$, where water confined in Nafion has been supposed to undergo a dynamical transition [13].

\section{Materials and methods}

\subsection{Materials and preparation of samples}

Nafion 115 membranes, with thickness of $\approx 127 \mu \mathrm{m}$ and equivalent weight of $1100 \mathrm{~g} /(\mathrm{mol}$ sulfonic group), were purchased from Aldrich. To remove impurities the membranes were boiled 1 hour in $3 \% \mathrm{H}_{2} \mathrm{O}_{2}, 1$ hour in $0.5 \mathrm{M} \mathrm{H}_{2} \mathrm{SO}_{4}$ and 1 hour in deionised $\mathrm{H}_{2} \mathrm{O}$. In all treatments the ratio (membrane mass, g) / (liquid volume, $\mathrm{mL}$ ) was $1 / 100$. After the first and the second treatment the membranes were rinsed in deionised water. The membranes were then wiped with a filter paper and cut so as to obtain $(5 \mathrm{~cm} \times 2 \mathrm{~cm})$ rectangular strips. After repeated rinsing in deionised water or in $\mathrm{D}_{2} \mathrm{O}$, the strips were stored at $58 \%$ relative humidity over saturated solutions of $\mathrm{NaBr}$ in $\mathrm{H}_{2} \mathrm{O}$ or $\mathrm{D}_{2} \mathrm{O}$, respectively. The $\mathrm{H}_{2} \mathrm{O}$ or $\mathrm{D}_{2} \mathrm{O}$ content was $0.092 w$ and $0.105 w$, corresponding respectively to $\lambda=5.6$ for $\mathrm{H}_{2} \mathrm{O}$ and 5.8 for $\mathrm{D}_{2} \mathrm{O}$. 


\subsection{Neutron scattering}

Measurements were made on the cold neutron multi-chopper time-of-flight spectrometer IN5 at the Institut Laue Langevin (ILL). From the collected intensity it is easy to derive the so-called dynamical structure factor $S(2 \theta, \omega)$ as a function of the scattering angle $2 \theta$ and the exchanged energy $\hbar \omega$ [15]. The dynamical structure factor $S(q, \omega)$ at a fixed exchanged momentum value $\hbar q$ can be derived from $S(2 \theta, \omega)$ by interpolation of the experimental data, taking into account the kinematical law that involves $q, 2 \theta$ and $\omega$. As the present sample can be considered isotropic, the dynamical structure factor depends effectively only on the modulus $q$ of the wave-vector transfer. An incident wavelength $\lambda=5 \AA$ was employed, achieving a $q$-range from $0.2 \AA^{-1}$ to $2.3 \AA^{-1}$ and an energy resolution with a full width at half maximum of $\sim 0.09 \mathrm{meV}$. The standard slab-shaped aluminum cell with a thickness of $0.5 \mathrm{~mm}$, has been placed into the neutron flux with an angle of $135^{\circ}$ with respect to the incident beam. The raw spectra were corrected for empty cell contribution, self-shielding and self-absorption, and normalized to a vanadium standard. Then the spectra were binned into 27 constant angle spectra. Due to the high value of the transmission coefficient $\left(t\left(90^{\circ}\right)=0.95\right)$ multiple scattering has been neglected. The samples have been investigated by warming up them in the temperature range from $200 \mathrm{~K}$ to $300 \mathrm{~K}$.

[Insert Fig. 1 about here]

\section{Results and discussion}

The signal of $\mathrm{H}_{2} \mathrm{O}$ - and $\mathrm{D}_{2} \mathrm{O}$-hydrated Nafion membrane can be described as it follows:

$$
\begin{aligned}
& S_{\text {Nafion }+\mathrm{H}_{2} \mathrm{O}}(2 \theta, \omega)=S_{\text {Nafion }}(2 \theta, \omega)+S_{\mathrm{H}_{2} \mathrm{O}}(2 \theta, \omega) \\
& S_{\text {Nafion }+D_{2} \mathrm{O}}(2 \theta, \omega)=S_{\text {Nafion }}(2 \theta, \omega)+S_{D_{2} \mathrm{O}}(2 \theta, \omega)
\end{aligned}
$$

The first term in the right side of the equations above, $S_{\text {Nafion }}(2 \theta, \omega)$, can be supposed to contribute to the elastic and the inelastic parts of the spectra, since the measurements have been done well below the glass transition temperature of the Nafion membrane $>370 \mathrm{~K}$ [16]. On the other hand, the second term in the right side of Eq. $1 \mathrm{a}$ and $1 \mathrm{~b}, S_{\mathrm{H}_{2} \mathrm{O}}(2 \theta, \omega)$ and $S_{D_{2} \mathrm{O}}(2 \theta, \omega)$, should contribute to the elastic, the quasielastic and the inelastic signal, as a consequence of the diffusive dynamics of the water molecules confined within the membrane. In Eq. 1a the incoherent contribution to the total cross sections from $S_{\mathrm{H}_{2} \mathrm{O}}(2 \theta, \omega)\left(\sigma_{\text {inc }}=959 \mathrm{~b}\right.$ and $\left.\sigma_{\mathrm{coh}}=46 \mathrm{~b}\right)$ is larger than that from the Nafion membrane $S_{\text {Nafion }}(2 \theta, \omega)$, which is purely coherent $\left(\sigma_{\mathrm{coh}}=290 \mathrm{~b}\right.$ per monomer). On the other hand, in Eq. $1 \mathrm{~b}$ the main contribution comes out from the coherent signal of the Nafion membrane with respect to heavy water $S_{D_{2} O}(2 \theta, \omega)\left(\sigma_{\text {inc }}=25 \mathrm{~b}\right.$ and $\left.\sigma_{\text {coh }}=93 \mathrm{~b}\right)$. The subtraction of the contribution to the signal due to the Nafion membrane has been done at each temperature through the relationship:

$$
S_{\mathrm{H}_{2} \mathrm{O}}(2 \theta, \omega) \approx S_{\mathrm{Nafion}+\mathrm{H}_{2} \mathrm{O}}(2 \theta, \omega)-S_{\mathrm{Nafion}_{\mathrm{D}} \mathrm{O}} \mathrm{O}(2 \theta, \omega)
$$

The difference spectra obtained with Eq. 2 are reported in Fig. 1 at 200K and 300K. The elastic intensity of the room temperature sample is lower than that at low-temperature, thus suggesting that hydrogen atoms are more mobile at $300 \mathrm{~K}$. This is consistent with both a decreasing Debye-Waller factor $\exp \left(-q^{2}<u(T)^{2}>\right)$, where $<u(T)^{2}>$ are the proton mean square displacements, and a more marked decay of the EISF with the temperature. In fact, also the increase of the quasielastic signal from $200 \mathrm{~K}$ to $300 \mathrm{~K}$, especially in the region below $2 \mathrm{meV}$, suggests that the hydrogen diffusive motions are activated as temperature increases. The features of the inelastic part are quite depressed, due to the strong decrease of the dynamical structure factor as a function of the energy. Then, to 
emphasize the inelastic trend as a function of the energy, a more suitable spectral representation is that of the so-called dynamical susceptibility $\chi(q, \omega)=S(q, \omega) / n(\omega, T)$, where $n(\omega, T)=\left(1-\exp \left(\hbar \omega / K_{B} T\right)\right)$ is the Bose occupation factor. Such factor removes the temperature dependence in the one-phonon approximation, provided that the system is harmonic, thus providing precious information on the harmonic or anharmonic character of the spectral features. $\chi(q, \omega)$ at a constant value of $q=1 \AA^{-1}$ has been directly estimated from $S(q, \omega)$ by interpolation or extrapolation, by supposing a $q^{2}$ trend for the $S(\mathrm{q}, \omega)$ wavevector dependence [15].

\section{[Insert Fig. 2 about here]}

In Fig. 2 we show the dynamical susceptibility of water confined in Nafion at $200 \mathrm{~K}$ and $300 \mathrm{~K}$. At room temperature four distinct features are well visible: a bump at around $1 \mathrm{meV}$, an inelastic peak at approximately $7 \mathrm{meV}$, a shoulder at $\sim 25 \mathrm{meV}$ and a rise at about $40 \mathrm{meV}$. This behaviour is quite similar to that of bulk water at $300 \mathrm{~K}$, whose dynamical susceptibility as measured by Raman scattering is displayed in the inset of Fig. 2 [17]. The peak at $\sim 7 \mathrm{meV}$ is attributed to collective transverse acoustic modes of the hydrogen bond network, i.e. the $\mathrm{O}-\mathrm{O}-\mathrm{O}$ bending-like motion of oxygen atoms perpendicular to the line formed by the hydrogen bond $\mathrm{O}-\mathrm{H} \cdots \mathrm{O}$ [18]. On the other hand, collective longitudinal acoustic modes, i.e. the $\mathrm{O}-\mathrm{O}-\mathrm{O}$ stretching-like motion of oxygen atoms along the line formed by the hydrogen bond $\mathrm{O}-\mathrm{H} \cdots \mathrm{O}$, would be at the origin of the shoulder at around $25 \mathrm{meV}$ [18]. The rise at about $40 \mathrm{meV}$ is instead attributed to librational motions of water molecules [18]. The physical meaning of the bump at approximately $1 \mathrm{meV}$ is quite debated. Some authors hypothesize that the vibrational excitations of clusters of water molecules is at the origin of this feature [19]. In an alternative explanation the excess of scattering is related to phononfracton crossover for vibrations in clusters of water molecules [20]. More recently it has been shown that a Mode Coupling Theory approach is able to describe the dynamical spectrum of water and its temperature dependence [17]. In this framework the mode at $\sim 1 \mathrm{meV}$ was interpreted as the manifestation of the primary $\alpha$-relaxation process.

\section{[Insert Fig. 3 about here]}

Quite interestingly, this very low-frequency feature is markedly temperature-dependent, as it is illustrated in detail in Fig. 3. At low temperature the bump at $1 \mathrm{meV}$ is missing, while a deep minimum is well visible in the susceptibility, thus suggesting that the structural relaxations of water are noticeably slowed down. When the temperature increases, the contribution of low-frequency excitations or relaxations begins to fill the minimum and the bump appears gradually as a shoulder of the more defined peak at $7 \mathrm{meV}$. This peak shows a definitively harmonic character, i.e. its intensity is temperature independent, while the shoulder at $\sim 25 \mathrm{meV}$ seems to depend on the temperature, even if the statistics of the present experiment does not allow a detailed analysis of the relevant trend. Above $260 \mathrm{~K}$ the mode at $1 \mathrm{meV}$ is clearly visible and depends only weakly on the temperature. This behaviour seems to indicate that the activation of the relaxational degrees of freedom related with the feature at around $1 \mathrm{meV}$ takes place mainly in the temperature range below $\sim 260 \mathrm{~K}$. On the other hand, above $\sim 260 \mathrm{~K}$ the temperature dependence of these low-frequency processes is rather weak. In fact, both NMR and quasielastic neutron scattering investigations suggest that water confined in Nafion membranes undergoes a dynamical transition at $260 \mathrm{~K}[13,21]$. In a NMR experiment it was shown that water absorbed in Nafion is characterised by a high rotational mobility with low hydrogen bond

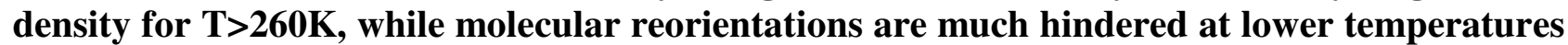
[13]. Very recently a quasielastic neutron scattering investigation pointed out that the typical jumping time of the diffusion dynamics of hydrogen atoms around the sulfonic sites is stable at $5.3 \mathrm{ps}$ up to $\sim 260 \mathrm{~K}$ and then abruptly decreases for higher temperatures [21]. Therefore, we may speculate that at the low hydration conditions of the present experiment and at 
temperatures lower than $\sim 260 \mathrm{~K}$ we are in presence of supercooled water with a high degree of amorphization due to both confinement and the interaction with ionic species, such as sulfonic groups, Zundel and Eigen ions. When temperature is raised, such a very coordinated water undergoes a melting-like transition, where new orientational degrees of freedom are available, due to the relaxational mechanisms of the hydrogen-bond network. The character of water absorbed in Nafion membrane seems to be intermediate between ice and bulk water. We may speculate that the onset at around $260 \mathrm{~K}$ is related to the behaviour of the proton conductivity of Nafion at about $0.08 w$, which shows a marked discontinuity between the low- and the hightemperature range near $260 \mathrm{~K}$ [22]

\section{Acknowledgements}

The Institut Laue-Langevin is kindly acknowledged for providing beam time and technical and scientific support. The authors are grateful to Dr. M. Plazanet and Dr. A. Orecchini for the assistance during the experiment on the IN5 spectrometer. 


\section{References}

[1] R. Bergman, J. Swenson, Nature 403283 (2000).

[2] V. Crupi, D. Majolino, P. Migliardo, V. Venuti, U. Wanderlingh, T. Mizota, M. Telling, J. Phys. Chem. B 1084314 (2004).

[3] B. Webber, J. Dore, J. Phys.: Cond. Matter 16 S5449 (2004).

[4] H. K. Christenson, J. Phys.: Cond. Matter 13 R95 (2001).

[5] M. Arndt, R. Stannarius, W. Gorbatschow, F. Kremer, Phys. Rev. E 545377 (1996).

[6] G. Carini, V. Crupi, G. D’Angelo, D. Majolino, P. Migliardo, Y. B. Mel'nichenko, J. Chem. Phys. 1072292 (1997).

[7] T. A. Zawodzinski, M. Neeman, L. O. Sillerud, S. Gottesfeld, J. Phys. Chem. 956040 (1991).

[8] K.-D. Kreuer, J. Membr. Sci. 18529 (2001).

[9] K. A. Mauritz, R. B. Moore, Chem. Rev. 1044535 (2004).

[10] T. D. Gierke, G. E. Munn, F. C. Wilson, J. Polym. Sci Polym. Phys. Ed. 191687 (1981).

[11] L. Rubatat, G. Gebel, O. Diat, Macromolecules 377772 (2004).

[12] A. M. Pivovar, B. S. Pivovar, J. Phys. Chem. B 109785 (2005).

[13] B. MacMillan, A. R. Sharp, R. L. Armstrong, Polymer 402471 (1999).

[14] M. Escoubes, M. Pineri, in Perfluorinated Ionomer Membranes, Edited by A. Eisenberg (ACS Symposium Series 110, Washinton DC, 1982).

[15] M. Bée, Quasi-elastic neutron scattering: principles and application in solid-state chemistry, biology, and materials science, Edited by A. Hilger (Bristol: Philadelphia, 1988).

[16] Y. S. Kim, L. Dong, M. A. Hickner, T. E. Glass, V. Webb, J. E. McGrath, Macromolecules 36 6281 (2003).

[17] A. P. Sokolov, J. Hurst, D. Quitmann, Phys. Rev. E 5112865 (1995).

[18] G. E. Walrafen, M. R. Fischer, M. S. Hokmabadi, W. H. Yang, J. Chem. Phys. 856970 (1986).

[19] J. L. Rousset, E. Duval, A. Boukenter, J. Chem. Phys. 922150 (1990).

[20] D. Majolino, F. Mallamace, P. Migliardo, et al., Phys. Rev. E 472669 (1993).

[21] A. Paciaroni, M. Casciola, E. Cornicchi, M. Marconi, G. Onori, M. Pica, R. Narducci, J. Chem. Phys. B 11013769 (2006).

[22] M. Cappadonia, W. E. Erning, S. M. S. Niaki, U. Stimming, Solid State Ionics 7765 (1995). 


\section{Figure captions}

Figure 1. Dynamical structure factor of $\mathrm{H}_{2} \mathrm{O}$ absorbed in Nafion at 200K (Empty circles) and 300K (Filled circles) at an average angle $2 \theta=65^{\circ}$. The dashed line is the energy resolution.

Figure 2. Dynamical susceptibility of water absorbed in Nafion at 200K (Empty circles) and 300K (Filled circles) at $\mathrm{Q}=1 \AA^{-1}$. Inset: dynamical susceptibility of bulk water at $300 \mathrm{~K}$ as measured by Raman scattering [17].

Figure 3. Dynamical susceptibility of water absorbed in Nafion at 200K (Empty circles), 230K (Filled circles), 260K (Empty upward triangles), 270K (Empty squares), 280K (Empty downward triangles), 290K (Stars), 300K (Filled triangles). 


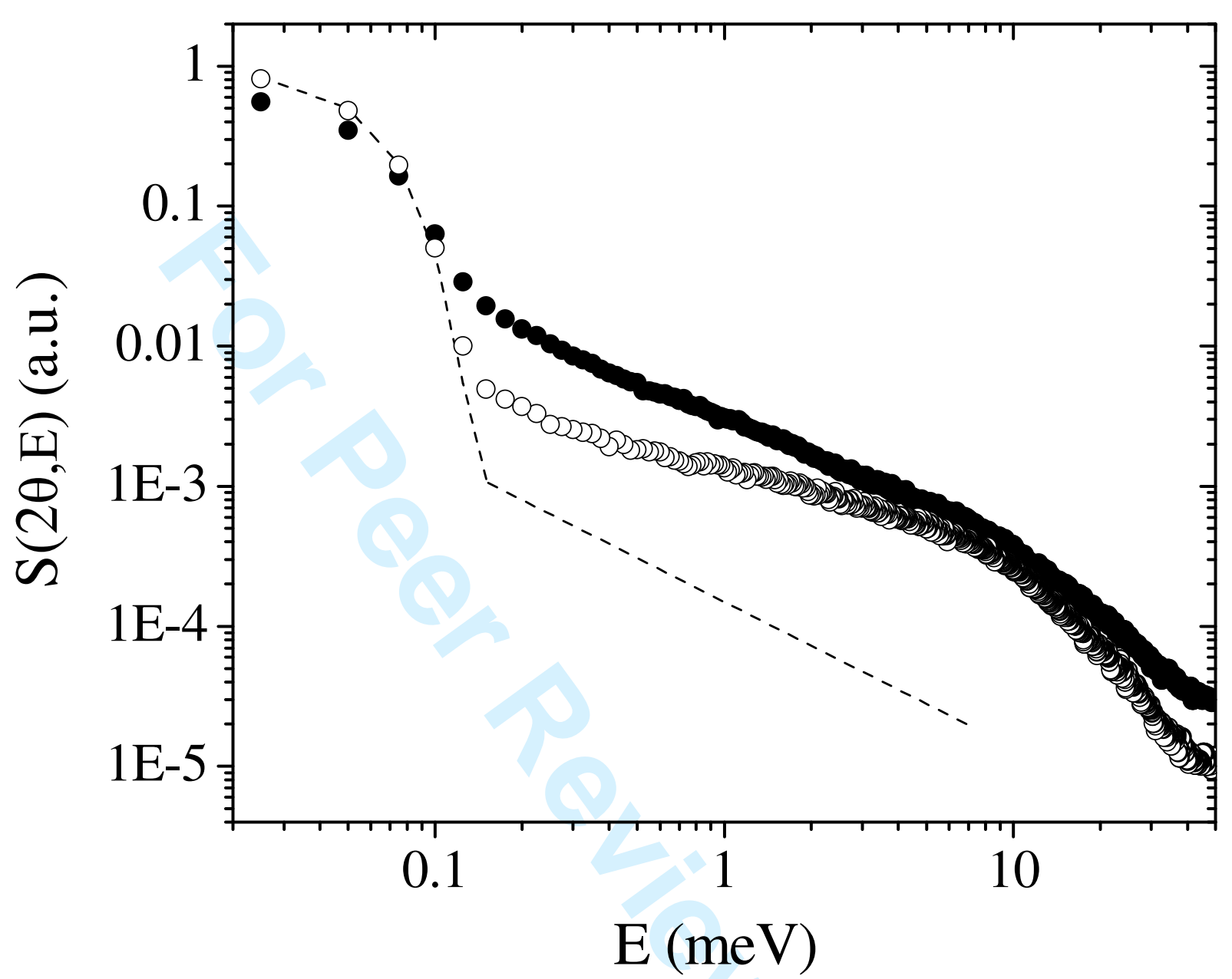

FIGURE 1 


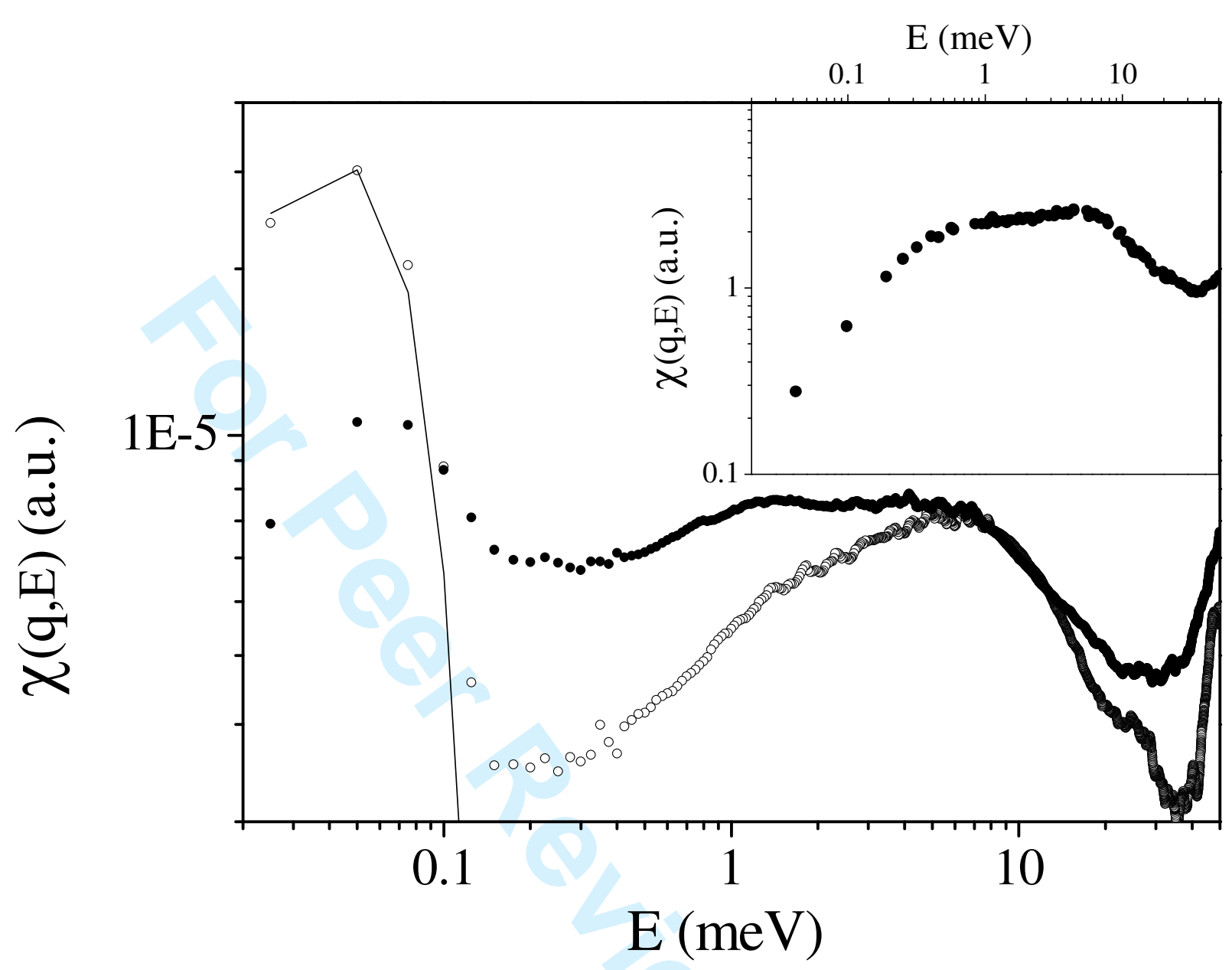

FIGURE 2 


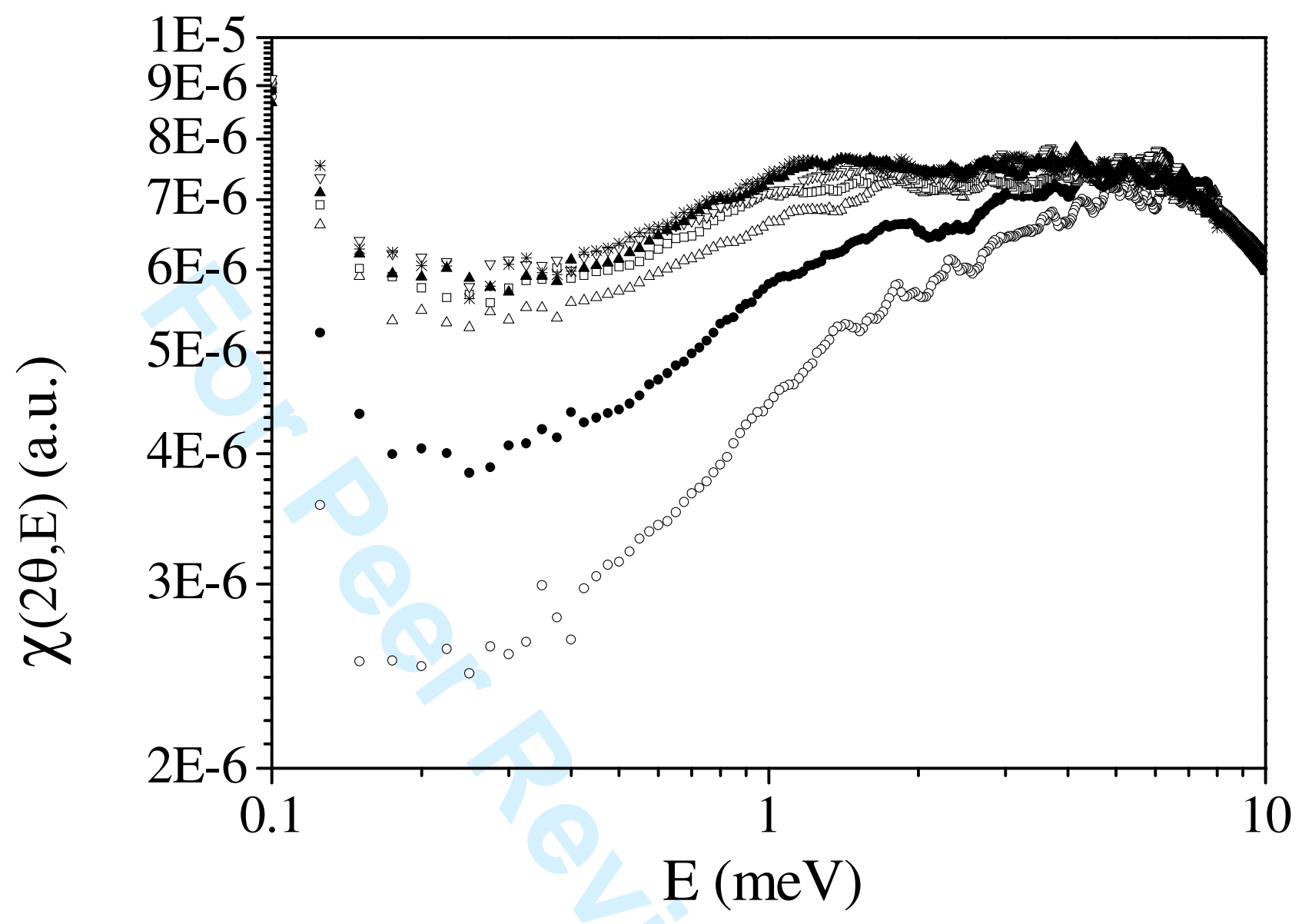

FIGURE 3 